\title{
Glycosylation of human adiponectin affects its conformation and stability
}

\author{
Philip W Peake, Jaquelyne T Hughes, Yvonne Shen and John A Charlesworth
}

Division of Medicine, Prince of Wales Hospital, Randwick, New South Wales 2031, Australia

(Requests for offprints should be addressed to P W Peake; Email: p.peake@unsw.edu.au)

\begin{abstract}
The collagenous region of adiponectin is glycosylated in vitro with glucosylgalactosyl moieties on four conserved lysines. We investigated the glycosylation of human adiponectin in vivo. Sugar vicinyl hydroxides on adiponectin were oxidized with 10 or $1 \mathrm{mM}$ metaperiodate, and the result analyzed by two-dimensional electrophoresis and immunoblotting. Only $10 \mathrm{mM}$ metaperiodate caused significant changes in electrophoretic mobility and an altered susceptibility to proteinase $\mathrm{K}$ digestion. Such treatment also increased the susceptibility of hexamers and high molecular weight (HMW) isoforms to dissociation by SDS. By contrast, untreated low molecular weight (LMW) isoforms were readily dissociated by low concentrations of SDS. Reduced HMW isoforms were able to partially reassemble following the removal of dithiothreitol, and this process was unaffected by metaperiodate. The presence of sialic acid was detected by Maackia amurensis Lectin II blotting, and by oxidation with $1 \mathrm{mM}$ metaperiodate, followed by detection with Emerald Green 300 fluorescent dye. Quantitation of sugars on affinity-purified adiponectin from nine human plasmas showed that dimers of HMW isoforms contained a 1.3-fold greater amount of total sugar than LMW isoforms. However, both contained similar amounts of sialic acid. We conclude that glucosylgalactosyl residues contribute to the conformation of HMW human plasma adiponectin. In addition, the HMW isoform contains greater amounts of glucosylgalactosyl residues than the LMW isoform, and these sugars are important in determining its stability in vivo.
\end{abstract}

Journal of Molecular Endocrinology (2007) 39, 45-52

\section{Introduction}

Adiponectin is an adipocyte-specific protein related in structure to the complement component Clq. As with C1q, it self-associates into trimers, as well as higher molecular weight isoforms. Depending upon the assay system employed, adiponectin has been shown to have insulin sensitizing, anti-atherogenic, and anti-inflammatory properties, and it interacts with a range of other proteins, including growth factors (Wang et al. 2005), and with lipopolysaccharides (Peake et al. 2006). The activity of the protein may depend upon the molecular structure, with the high molecular weight (HMW) isoforms being associated with insulin sensitivity in man (Pajvani et al. 2004). In addition, mutations of adiponectin have been reported in diabetic patients in whom the protein shows impaired multimerization (Waki et al. 2003). The basic subunit consists of a collagenous N-terminal domain, and a C-terminal globular head; these subunits associate to form a trimer, the low molecular weight (LMW) complex. Adiponectin may also form sulfhydryl-dependent hexamers (medium molecular weight; MMW) and HMW isoforms containing nine dimers (Suzuki et al. 2007). These are stable in vitro and in vivo (Peake et al. 2005), and do not interconvert. However, the mechanisms which determine the production of these different forms of the molecule remain unclear.

A number of studies have investigated the role of post-translational modifications in the assembly of the HMW isoform. The collagenous region of adiponectin produced in vitro contains four conserved lysines that are both hydroxylated and glycosylated with a glucosylgalactosyl moiety (Wang et al. 2002, 2004, Richards et al. 2006). Also present in bovine and mouse plasma adiponectin is sialic acid, possibly on O-linked glycans (Sato et al. 2001). However, the function of this modification has not been determined.

Mutant forms of recombinant human adiponectin expressed in a variety of mammalian cell lines have shown the importance of the glucosylgalactosyl moiety. When the modified lysines are replaced with arginine residues, the molecule has impaired function and is unable to form the HMW isoforms associated with insulin-sensitizing activity (Wang et al. 2006b). In this study, we investigated the role of sugars in native adiponectin derived from human plasma, since it is recognized that the choice of expression vector or culture conditions can affect the glycosylation of recombinant proteins (Skibeli et al. 2001, Brooks 2004). Its role in maintaining the structure and conformation of the protein was determined by the gentle conversion of 
vicinyl hydroxides on sugars to aldehyde by metaperiodate. Destruction of glucosylgalactosyl residues, but not of sialic acid, led to an increased susceptibility to protease digestion and a weaker interaction between the subunits comprising the HMW and MMW isoforms. In addition, we examined the quantity of sugars present in LMW and HMW isoforms isolated from human plasma and showed that the HMW isoforms were glycosylated to a greater degree than LMW isoforms. This correlated with resistance to disruption by SDS of the HMW multimer.

\section{Materials and methods}

\section{Superose column chromatography}

Adiponectin from the plasma of a healthy male donor was resolved on an FPLC Superose 12 column (Pharmacia) equilibrated with PBS (Peake et al. 2005). Adiponectin levels in fractions were measured by commercial ELISA (R\&D, Minneapolis, MN, USA).

The ability of the isoforms of plasma adiponectin to reassociate following reduction to the trimer by dithiothreitol (DTT) was determined by pooling fractions 4 and 5 (HMW) and 10 and 11 (LMW) following FPLC analysis. These samples were made to $0 \cdot 1 \mathrm{M}$ DTT and incubated for $60 \mathrm{~min}$ at $37^{\circ} \mathrm{C}$, and then transferred into PBS by passage down a G25 column. After incubation for $60 \mathrm{~min}$ at $37^{\circ} \mathrm{C}$, followed by overnight incubation at $4{ }^{\circ} \mathrm{C}$, samples were reanalyzed by fast protein liquid chromatography (FPLC). In some experiments, samples were also reacted in the dark for $30 \mathrm{~min}$ with $10 \mathrm{mM}$ metaperiodate before overnight incubation and reanalysis by FPLC.

\section{Electrophoretic analysis of adiponectin in plasma}

Plasma proteins were analyzed by native electrophoresis (Peake et al. 2005). In selected experiments, individual lanes were excised and analyzed in the second dimension (2D) by native electrophoresis, or equilibrated at room temperature with non-reducing SDSPAGE buffer before 2D analysis. In other experiments, strips were incubated with $10 \mathrm{mM}$ metaperiodate for 30 min before 2D separation. Blots were probed with a monoclonal (BD Biosciences, Sydney, NSW, Australia) or rabbit polyclonal (Sigma) antibody to adiponectin. These were detected with an affinity-purified, peroxidase-conjugated antibody to globulin (Bio-Rad) followed by chemiluminescence. Films were scanned and analyzed by Bio-Rad Quantity One software.

\section{Enzyme digestion}

To investigate any effect on protein structure induced by the destruction of sugars containing vicinyl hydroxides, adiponectin was incubated for $30 \mathrm{~min}$ in the dark with either $0 \cdot 1 \mathrm{M}$ acetate buffer $(\mathrm{pH} 5 \cdot 5)$ or with the same buffer containing $10 \mathrm{mM}$ sodium metaperiodate. Samples were then buffer exchanged into PBS by passage down a G25 column, and incubated for $1 \mathrm{~h}$ at $37^{\circ} \mathrm{C}$ with dilutions of $20 \mathrm{mg} / \mathrm{ml}$ proteinase $\mathrm{K}$ (Sigma) in PBS. The resulting fragments were analyzed by non-reducing SDS analysis and blotting.

\section{Detection of glycosylation}

Adiponectin from nine normal human plasmas (five males) was separated by FPLC analysis as above into HMW and LMW isoforms (Peake et al. 2005). Adiponectin was immunoprecipitated with a monoclonal antibody (R\&D), and a polyclonal rabbit anti-mouse antibody (Dako, Glostrup, Denmark) conjugated to Sephadex (Pharmacia). The Sephadex beads were washed six times in cold PBS containing 1\% NP40 and $0 \cdot 1 \%$ SDS, before analysis by non-reducing SDS electrophoresis. The amount of adiponectin was determined by analysis of western blots probed with monoclonal anti-human adiponectin (BD). Total glycoprotein was detected by staining of gels with ProQ Emerald Green 300, following manufacturer's instructions (Molecular Probes, Invitrogen). Sialic acid residues were detected in an identical fashion, except that the gel was incubated in $1 \mathrm{mM}$ metaperiodate for $30 \mathrm{~min}$, as described in the ECL glycoprotein detection system (Amersham Life Science).

\section{Lectin chromatography}

To investigate the presence of sialic acid, HMW and LMW fractions of adiponectin were immunoprecipitated before analysis by non-reducing SDS electrophoresis and western blotting. Blots were probed with biotinylated $1 \mu \mathrm{g} / \mathrm{ml}$ Maackia amurensis Lectin II (Vector Labs, Burlingame, CA, USA) in Tris buffer $(\mathrm{pH} 7 \cdot 5)$ containing $0 \cdot 2 \mathrm{M} \mathrm{NaCl}$. Lectin was detected by an avidin-peroxidase conjugate and chemiluminescence. Blots were reprobed with a monoclonal antibody to confirm that lectin was bound to adiponectin.

\section{Statistical analysis}

Electrophoretic analyses were performed a minimum of three times; results for representative experiments are shown. Data shown on the quantity of sugars in the fractions of individual plasmas and the amount of adiponectin in FPLC fractions represent the mean of duplicate analyses. Comparisons between groups were performed using paired and unpaired $t$-tests or the Wilcoxon signed-rank test. $P$ values $<0.05$ were considered statistically significant. 


\section{Results}

\section{D gel electrophoresis}

As previously described (Peake et al. 2005), plasma adiponectin was resolved by native gel electrophoresis into four major bands (Fig. 1A); there were two HMW bands, as well as MMW, and LMW bands. A minor band was encountered in some samples, between the HMW and MMW isoforms. Incubation with $10 \mathrm{mM}$ metaperiodate led to an altered electrophoretic pattern, with an increased number of bands, most of which moved further than usual in the gel (Fig. 1A). Incubation with $1 \mathrm{mM}$ metaperiodate did not have any significant effect.
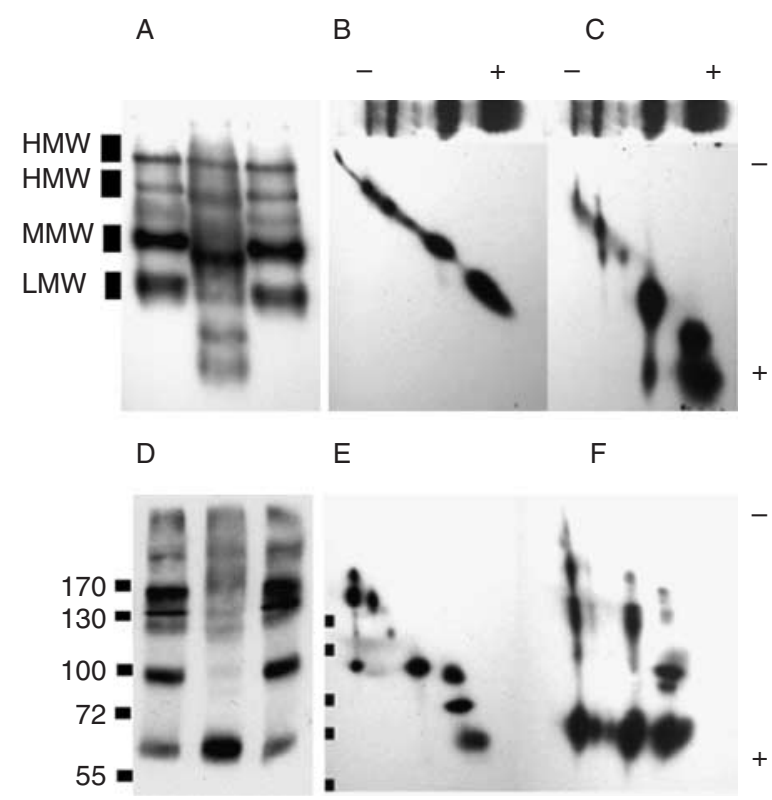

Figure 1 (A) A western blot, following native gel electrophoresis of plasma adiponectin. This shows the effect on adiponectin of incubation with acetate buffer (lane 1), acetate buffer containing $10 \mathrm{mM}$ metaperiodate (lane 2), and $1 \mathrm{mM}$ metaperiodate (lane 3). Two HMW bands are shown, as well as the MMW and LMW bands. ( $B$ and $C$ ) Western blots showing the separation of plasma adiponectin by native electrophoresis are shown as horizontal inserts at the top of $1 \mathrm{~B}$ and $1 \mathrm{C}$. Below these inserts is a western blot showing the separation of adiponectin by $2 \mathrm{D}$ (native gel/native gel) electrophoresis. Plasma was separated in the second dimension by native electrophoresis following incubation of gel strips from the first dimension with either acetate buffer (1B), or buffer containing $10 \mathrm{mM}$ metaperiodate (1C). (D) A western blot following non-reducing SDS gel electrophoresis showing the effect on plasma adiponectin of incubation with acetate buffer (lane 1), or acetate buffer containing 10 (lane 2) or $1 \mathrm{mM}$ (lane 3) metaperiodate. Molecular weight markers $(\mathrm{kDa})$ are shown at left. ( $E$ and $F$ ) A western blot following 2D (native gel/non-reducing SDS gel) separation of plasma adiponectin. Plasma was separated in the first dimension by native electrophoresis, followed by incubation of gel strips with either acetate buffer (1E), or buffer containing $10 \mathrm{mM}$ metaperiodate $(1 \mathrm{~F})$. These were separated in the second dimension by non-reducing gel electrophoresis in $0.4 \%$ SDS. The same molecular weight markers as in 1D are shown at left.
In non-reducing SDS gels, the pattern seen was unaffected by incubation with $1 \mathrm{mM}$ metaperiodate (Fig. 1D). However, incubation with $10 \mathrm{mM}$ metaperiodate led to a different pattern of bands, with a loss of many bands of high MW. There was an increased representation of the dimer of $\sim 65 \mathrm{kDa}$ (Fig. 1D).

\section{D gel electrophoresis}

We sought to clarify these results by 2D electrophoresis. Adiponectin was separated in the first dimension by native electrophoresis, followed by incubation of the gel with either buffer, or buffer containing $10 \mathrm{mM}$ metaperiodate, and subsequent 2D analysis by either native (Fig. 1B and C) or non-reducing SDS electrophoresis (Fig. $1 \mathrm{E}$ and $\mathrm{F}$ ).

\section{Native/native 2D gels}

Adiponectin incubated with buffer alone was unaffected in 2D analysis (Fig. 1B). However, incubation with $10 \mathrm{mM}$ metaperiodate produced species derived from HMW, MMW, and LMW isoforms that had greater electrophoretic mobility than the native isoform (Fig. 1C).

\section{Native/non-reducing SDS 2D gels}

Non-reducing analyses of native gel strips resolved the HMW isoforms into several bands $>170 \mathrm{kDa}$, but not into the dimer of $\sim 65 \mathrm{kDa}$. The LMW fraction was resolved into three bands, including the dimer (Fig. 1E). However, incubation with metaperiodate led to a different pattern for both the HMW and MMW isoforms (Fig. 1F). In both cases, there was extensive formation of the dimer, while the amount of dimer present in the LMW fraction was not significantly affected. Metaperiodate had little effect on the amount of dimer derived from LMW adiponectin, the proportion of which remained $0 \cdot 4-0 \cdot 5$ under a range of SDS concentrations (Fig. 2). By contrast, both HMW and MMW isoforms were far more susceptible to dissociation by SDS after incubation with metaperiodate than with acetate buffer alone. Incubation with SDS at $37^{\circ} \mathrm{C}$ rather than room temperature did not have a significant additional effect (not shown).

\section{Reassociation of HMW and LMW fractions after reduction with DTT}

Size-sorting analysis by FPLC showed that reduction converted all HMW adiponectin to the LMW trimer. However, on removal of DTT, the trimers derived from this fraction were able to substantially reassemble into species of HMW (Fig. 3). However, it was clear from Fig. 3, and from other analyses on native gels, that they 


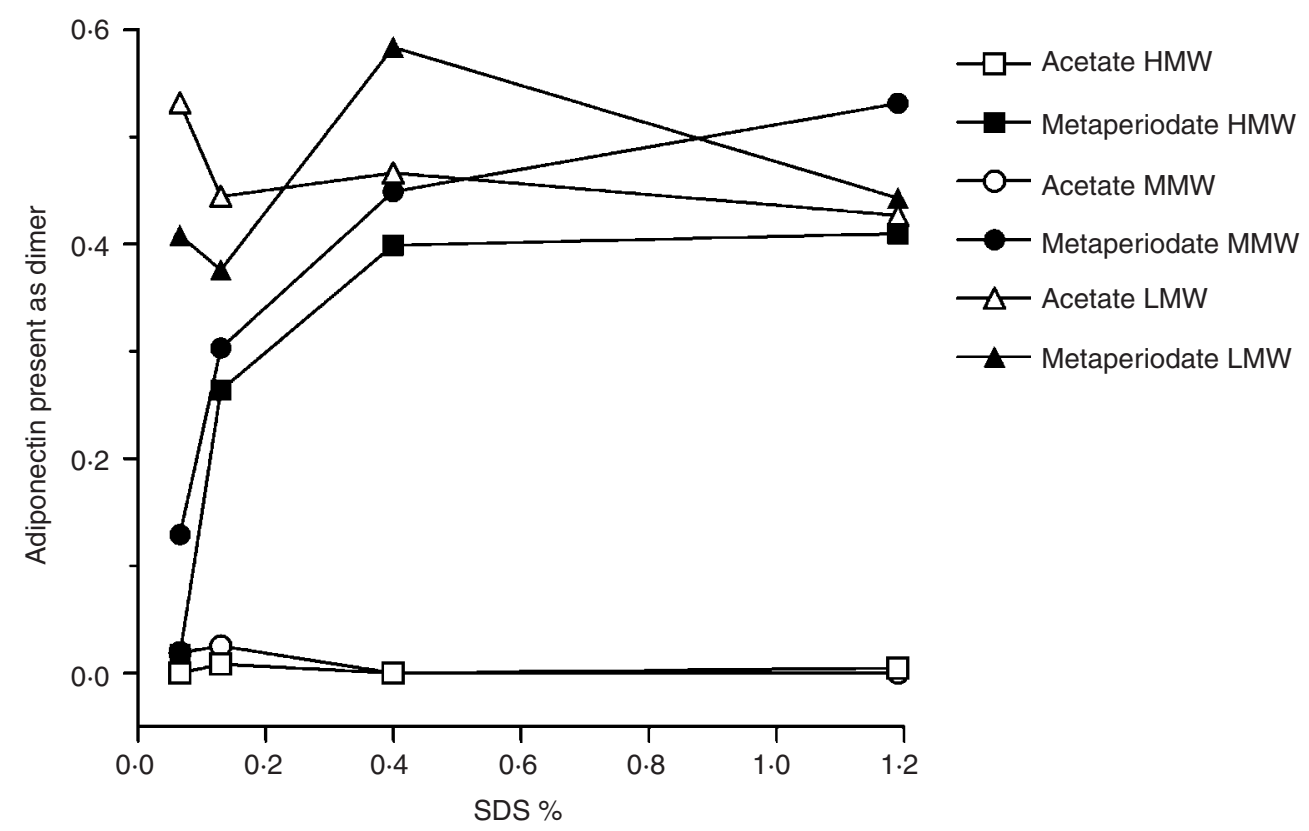

Figure 2 Effect of incubation with metaperiodate or acetate buffer on the quantity of adiponectin dimer in HMW, MMW, and LMW isoforms from plasma adiponectin. Plasma was separated by 2D (native gel/nonreducing SDS gel) electrophoresis, as shown in Fig. 1E and F. Before separation in the second dimension, gel strips were incubated in varying concentrations of SDS.

had not fully reassembled to the size of the HMW isoform present in plasma. On the other hand, LMW isoforms remained a similar size after removal of DTT.

When reduced fractions of adiponectin were additionally oxidized with metaperiodate, the same results were seen. There were no significant changes in the apparent sizes of HMW or LMW isoforms following oxidation with metaperiodate alone (not shown).

\section{Enzymatic digestion}

To further investigate the influence of glycosylation on the structure of adiponectin, the effect of incubation with metaperiodate on the susceptibility of adiponectin to enzymatic digestion by proteinase $\mathrm{K}$ was evaluated (Fig. 4A). Adiponectin was present as a dominant band of $\sim 65 \mathrm{kDa}$ consistent with the dimer, along with another band of $\sim 24 \mathrm{kDa}$. On incubation with increasing concentrations of proteinase $\mathrm{K}$, there were clear differences between adiponectin samples that had or had not been preincubated with $10 \mathrm{mM}$ metaperiodate. Samples treated with $10 \mathrm{mM}$ metaperiodate showed the appearance of a major breakdown product of $\sim 35 \mathrm{kDa}$ not seen in samples preincubated in buffer alone. Similarly, there was an increased susceptibility of metaperiodatetreated adiponectin to digestion by $0.05 \mathrm{mg} / \mathrm{ml}$ enzyme in terms of the formation of a band of $\sim 20 \mathrm{kDa}$, and in terms of the amount of adiponectin remaining as a dimer. Plasmas similarly treated after incubation with $1 \mathrm{mM}$ metaperiodate showed no differences to those treated with buffer alone (not shown).

\section{Glycosylation of adiponectin}

The presence of sialic acid on human adiponectin was strongly suggested by the ability of Maackia amurensis Lectin II to bind to affinity-purified HMW and LMW adiponectin (Fig. 4B). This was confirmed by the staining of polyacrylamide gels with ProQ Emerald 300 stain after oxidation with $1 \mathrm{mM}$ metaperiodate (Fig. 5C) rather than $44 \mathrm{mM}$ metaperiodate employed to detect total glycoprotein (Fig. 5B).

Recombinant adiponectin was of slightly higher MW than affinity-purified human adiponectin (Fig. 5A), and equivalent amounts of this protein, as determined by analysis of antigenic blots and gels stained with ProQ Emerald 300, showed an increased degree of glycosylation. Both HMW and LMW fractions were glycosylated, and both contained vicinyl hydroxides able to be oxidized by $1 \mathrm{mM}$ metaperiodate.

Quantitative analysis of the intensity of Emerald 300 staining (Fig. 5B and $\mathrm{C}$ ) with reference to the intensity of antigenically detected adiponectin (Fig. 5A) showed that staining intensity was proportional to the quantity of adiponectin present, and that both HMW and LMW fractions contained lesser amounts of total sugar and sialic acid than the recombinant molecule. There were no significant differences between repeated assays of 


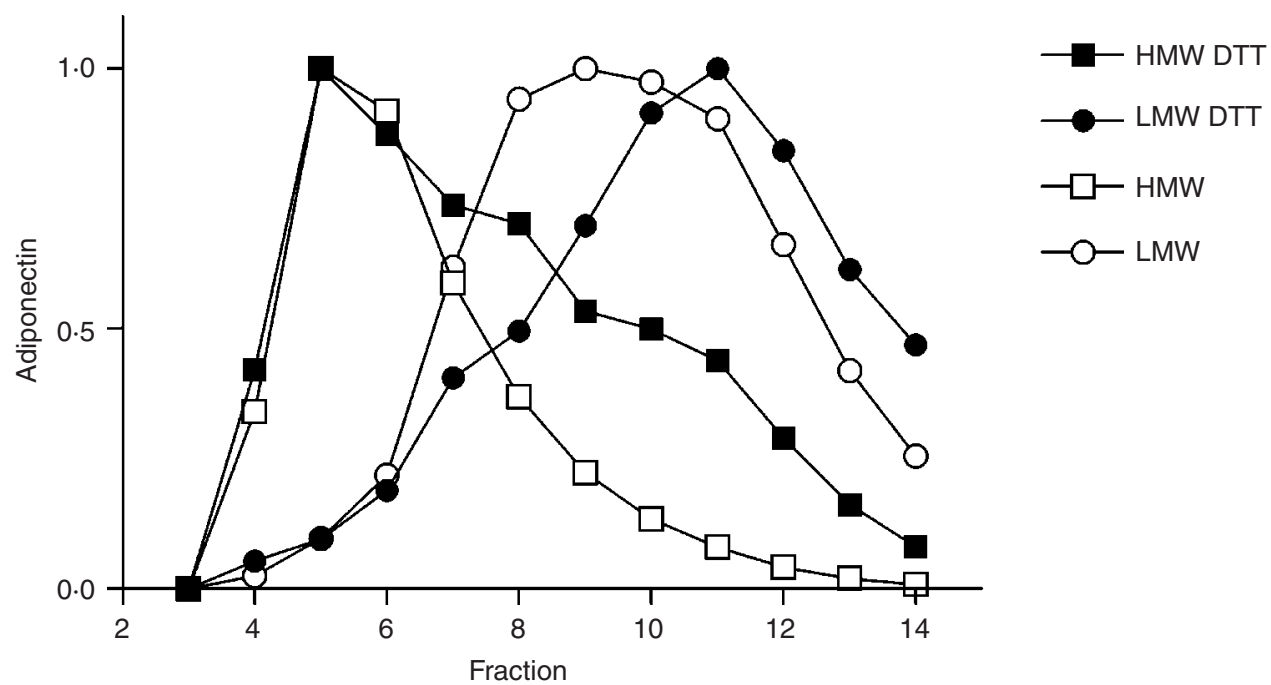

Figure 3 FPLC size-sorting analyses of HMW and LMW fractions of adiponectin derived from human plasma. These HMW and LMW fractions were incubated with PBS (open symbols) or DTT (closed symbols). This was then removed before further incubation with PBS. These samples were then reanalyzed by size-sorting FPLC. Data from each analysis were normalized to the highest concentration of adiponectin detected in the individual fractions of each FPLC analysis ( $y$ axis). The $x$ axis shows individual fractions from the FPLC column.

the same plasmas, and the S.E.M. of repeated observations was $\pm 11 \%$.

A paired-comparison analysis in nine individual normal plasmas revealed that the total amount of glycosylation in the HMW fractions was significantly greater than that in the LMW fractions $(P=0 \cdot 01)$, although there was no significant difference between levels of sialic acid in these fractions (Fig. 6A). The ratio of the levels of sugars in the HMW to the LMW fraction for these plasmas (Fig. 6B) was $1 \cdot 32 \pm 0 \cdot 10$ for total sugars, which was significantly greater than the expected 'null' value of $1 \cdot 0(P=0 \cdot 01)$. There were no significant differences in this ratio for the amount of sialic acid $(1 \cdot 18 \pm 0 \cdot 12)$.

\section{Discussion}

Different isoforms of adiponectin activate different signaling pathways and have differing effects in a range of tissues (Tsao et al. 2002, 2003, Wang et al. 2005). The data in this report emphasize the importance of glycosylation to the structure and conformation of these isoforms in human plasma. The distinction between recombinant forms of the molecule and those found in the plasma is important, since it is recognized that the choice of expression vector or culture conditions can affect the glycosylation of recombinant proteins (Brooks 2004). We showed recombinant human adiponectin to be of higher molecular weight, and more heavily glycosylated, than that present in human plasma.
The glucosylgalactosyl-modified lysines on the collagenous portion of adiponectin detected in vitro are presumed to be those present in vivo (Wang et al. 2002, 2004, Richards et al. 2006). Sialic acid has also been detected, but only in adiponectin purified from bovine and mouse plasma (Sato et al. 2001). Our data identify that sialic acid is present in equal amounts on HMW and LMW isoforms derived from human plasma. The analysis of total glycosylation revealed that dimers of the HMW isoform had 1.3 times the glycosylation present in the LMW isoform. Since this figure would include both glucosylgalactosyl and sialic acid residues, the true ratio of glucosylgalactosyl residues in HMW versus LMW isoforms is likely to be much greater than $1 \cdot 3$. This conclusion is supported by a study of unseparated plasma adiponectin in type 2 diabetic patients, in whom glycosylation of adiponectin was reduced in comparison with adiponectin from healthy controls. This may well have reflected a parallel fall in the proportion of the HMW isoforms (Wang et al. $2006 b$ ), shown by our results to have a greater amount of sugar than LMW isoforms.

Selective oxidation of sialic acid with $1 \mathrm{mM}$ metaperiodate allowed Emerald 300 dye binding. However, only incubation with $10 \mathrm{mM}$ metaperiodate had any effect on adiponectin's electrophoretic mobility, or upon the bands yielded on digestion with proteinase $\mathrm{K}$. This effect was likely to be related to the conformation of the protein, rather than any effect on charge. Metaperiodate oxidizes vicinyl hydroxyl groups on sugars to form aldehydes, and while $1 \mathrm{mM}$ metaperiodate was sufficient to oxidize sialic acid in readily detectible 


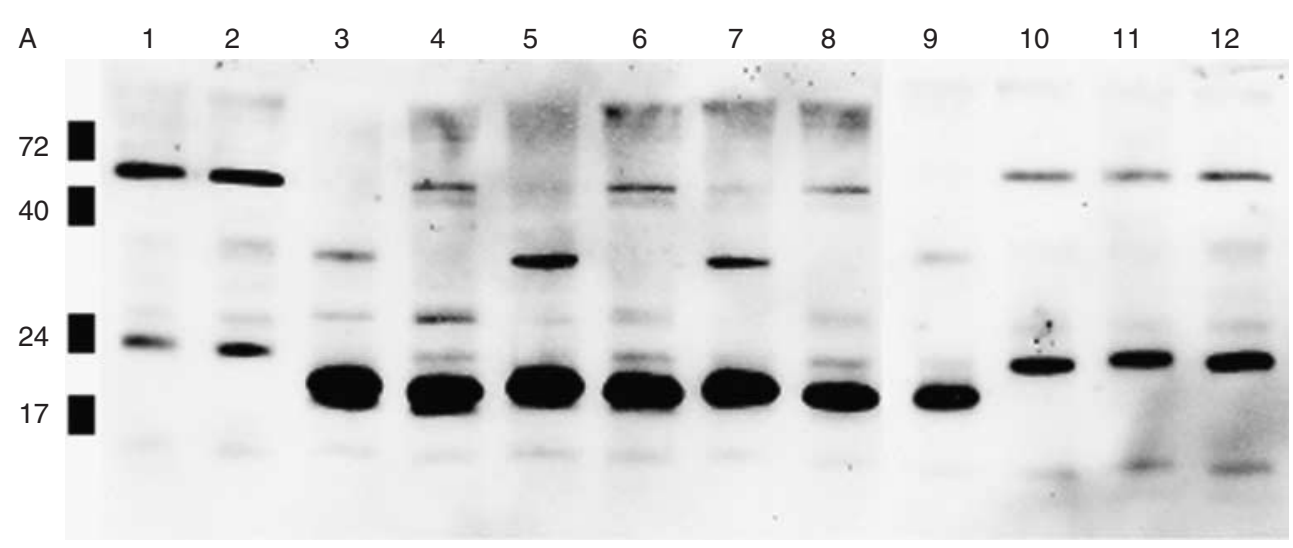

B

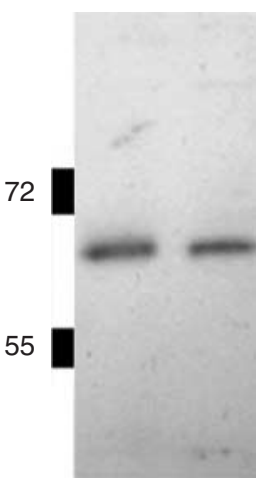

Figure 4 (A) A western blot following non-reducing SDS gel electrophoresis showing the effect of incubation with $10 \mathrm{mM}$ metaperiodate on the cleavage of adiponectin by proteinase $\mathrm{K}$. Plasmas were incubated in buffer containing metaperiodate, or buffer alone. Following this treatment, samples were re-equilibrated with PBS, and then incubated for $60 \mathrm{~min}$ at $37^{\circ} \mathrm{C}$ with proteinase $\mathrm{K}$. The cleavage pattern generated by proteinase $\mathrm{K}$ on samples preincubated with metaperiodate (odd lane numbers), or buffer alone (even lane numbers) is shown. Proteinase $\mathrm{K}$ was used at concentrations of 0 (lanes 1 and 2), 1.30 (lanes 3 and 4), 0.44 (lanes 5 and 6), 0.15 (lanes 7 and 8), 0.05 (lanes 9 and 10), or 0.0015 mg/ml enzyme (lanes 11 and 12). Molecular weight markers are shown at left. (B) A western blot following non-reducing SDS gel electrophoresis showing the binding of Maackia amurensis Lectin II, which has specificity for sialic acid, to affinity-purified HMW (lane 1) and LMW (lane 2) fractions of adiponectin. Molecular weight markers $(\mathrm{kDa})$ are shown at left.

quantities, it failed to alter the electrophoretic distribution of adiponectin. Evidence supporting a change in conformation due to the destruction of glycosylated residues came with the demonstration that incubation with $10 \mathrm{mM}$ metaperiodate affected the pattern of cleavage generated by proteinase $\mathrm{K}$. This enzyme cleaves at the carboxyl side of hydrophobic, aliphatic, and aromatic amino acids, and changes to the cleavage pattern suggest alterations in the accessibility of some of these residues to the external environment.

The hexamer and HMW isoforms are mostly composed of disulfide-linked dimers, while the native trimer contains one monomer and one dimer, or three non-covalently associated monomers when reduced by DTT (Tsao et al. 2003). Our results show that once reduced, trimers derived from HMW isoforms tended to reassociate on removal of DTT, while the LMW trimer remained unchanged. This process was unaffected by oxidation of sugar residues and presumably depends upon non-covalent interactions between different subunits. The reassembly of trimers to HMW isoforms was incomplete, suggesting that glycosylation of the conserved lysine residues alone is insufficient for complete reconstitution of the HMW isoforms. Appropriate formation of disulfide bonds would appear crucial, since the HMW isoform contains nine disulfide-linked dimers (Tsao et al. 2003, Suzuki et al. 2007), implying that it could not be readily assembled from the trimer, which contains a dimer and a monomer with a single available sulfhydryl group. The process appears to require additional intracellular events, and evidence suggests that the HMW isoform is secreted via a pathway separate from that of other isoforms ( $\mathrm{Xu}$ et al. 2005). 
A

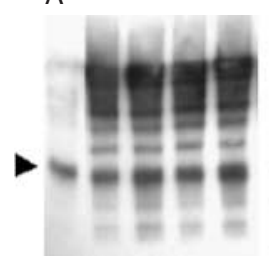

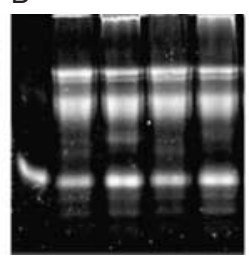

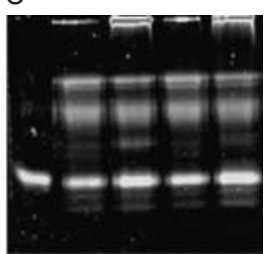

Figure 5 (A) A western blot following non-reducing SDS gel electrophoresis showing adiponectin antigenically detected by a monoclonal antibody. Recombinant adiponectin is shown in lane 1 (arrowhead), and affinity-purified HMW and LMW fractions are shown from two different plasmas in lanes 2 and 3 , and lanes 4 and 5 respectively. (B) A non-reducing SDS gel showing total sugars from HMW and LMW fractions of adiponectin detected by ProQ Emerald 300 after treatment with $44 \mathrm{mM}$ metaperiodate. Recombinant adiponectin is shown in lane 1 (arrowhead), and analyse of affinity-purified HMW and LMW fractions from the two plasmas in (A) are shown in lanes 2 and 3 , and lanes 4 and 5 respectively. (C) A non-reducing SDS gel treated showing sialic acid residues from HMW and LMW adiponectin detected by ProQ Emerald 300 after treatment with $1 \mathrm{mM}$ metaperiodate. Recombinant adiponectin is shown in lane 1 (arrowhead), and analyses of affinity-purified HMW and LMW fractions from the two plasmas in Fig. 5A are shown in lanes 2 and 3 , and lanes 4 and 5 respectively.

We found that LMW adiponectin readily dissociated to a dimer in the presence of SDS, irrespective of whether or not its carbohydrates had been oxidized, whereas the HMW and MMW isoforms were more stable. However, these became susceptible to disruption subsequent to complete oxidation of their carbohydrate moieties, but not when sialic acid was preferentially destroyed. Sugars are thought to increase the stability of the collagen helix (Bann $\&$ Bachinger 2000), and the glucosylgalactosyl-modified lysine residues may act cooperatively in the formation of HMW isoforms (Wang et al. 2006b). Since electron microscopy of the hexamer and HMW isoforms indicates that they contain a single stalk of collagen domains (Tsao et al. 2003, Suzuki et al. 2007), our data suggest that glucosylgalactosyl-modified lysines stabilize interactions between the collagenous regions of dimers within the HMW and MMW isoforms, but not between the dimer and monomer within the LMW trimer. Further investigation is needed to define the full range of variants in hydroxylation and glycosylation of plasma adiponectin, and the effect such modifications may have on receptor binding, and interactions with other proteins (Wang et al. 2006a).

In summary, we showed that sialic acid was present in human plasma adiponectin and identified differences between the HMW and LMW isoforms in their degree of glycosylation, and in the influence of glycosylation on their structure and stability. In particular, it was the glucosylgalactosyl moiety, rather than sialic acid, which was crucial in these roles. The HMW and LMW isoforms had the same amount of sialic acid, but the former had a greater degree of glycosylation, and thus of the glucosylgalactosyl moiety. In addition, destruction of

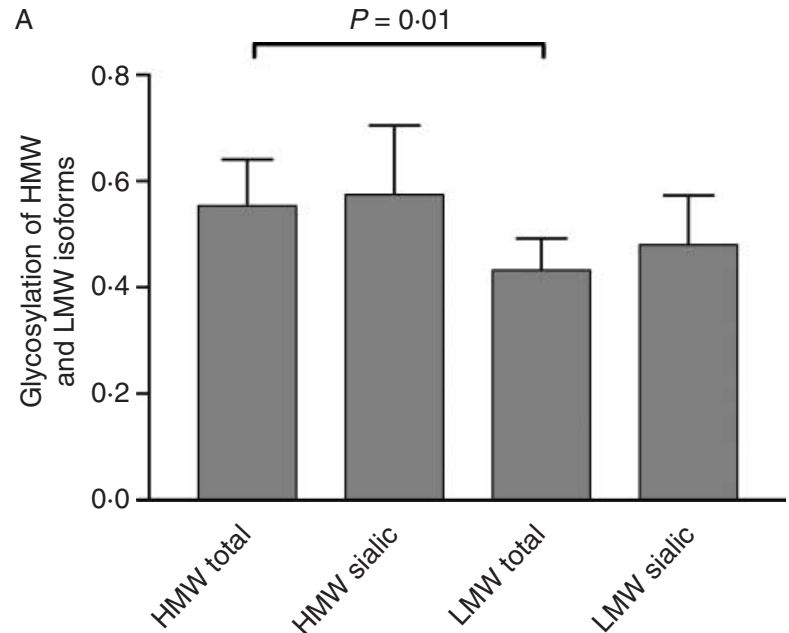

B

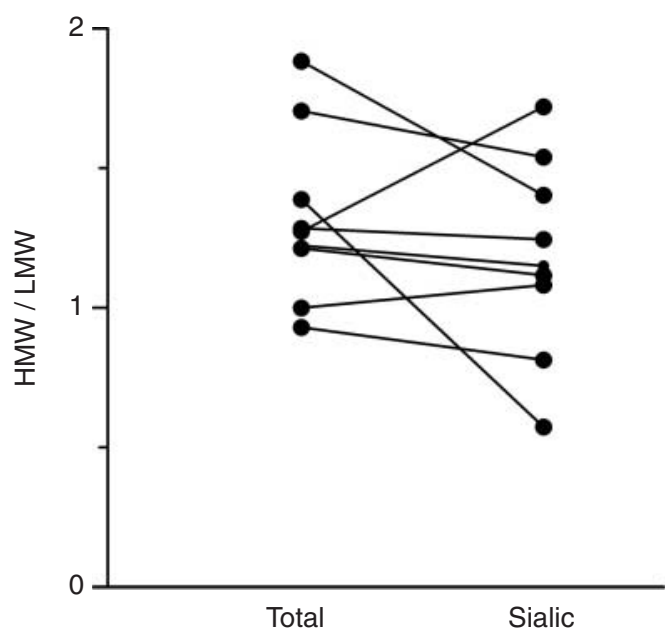

Figure $6(A)$ Levels of glycosylation of affinity-purified HMW and LMW fractions of plasma adiponectin. Gels were incubated with 44 or $1 \mathrm{mM}$ metaperiodate, to detect total glycosylation, or sialic acid respectively. Results are expressed relative to the glycosylation of recombinant adiponectin as the mean \pm S.E.M. (B) Levels of total sugars and sialic acid in affinity-purified HMW and LMW fractions of adiponectin from nine individual normal plasmas. Data are expressed as the ratio of the amount of sugars in HMW to LMW fractions in each plasma for both total sugars and sialic acid.

sialic acid had no effect on the stability or conformation of adiponectin, while modification of the glucosylgalactosyl moiety led to significant changes in the conformation of adiponectin and in the stability of the HMW and MMW complexes.

\section{Acknowledgements}

There is no conflict of interest that would prejudice the impartiality of these findings. 


\section{References}

Bann JG \& Bachinger HP 2000 Glycosylation/hydroxylation-induced stabilization of the collagen triple helix. 4-trans-hydroxyproline in the Xaa position can stabilize the triple helix. Journal of Biological Chemistry 275 24466-24469.

Brooks SA 2004 Appropriate glycosylation of recombinant proteins for human use: implications of choice of expression system. Molecular Biotechnology 28 241-255.

Pajvani UB, Hawkins M, Combs TP, Rajala MW, Doebber T, Berger JP, Wagner JA, Wu M, Knopps A, Xiang AH et al. 2004 Complex distribution, not absolute amount of adiponectin, correlates with thiazolidinedione-mediated improvement in insulin sensitivity. Journal of Biological Chemistry 279 12152-12162.

Peake PW, Kriketos AD, Campbell LV, Shen Y \& Charlesworth JA 2005 The metabolism of isoforms of human adiponectin: studies in human subjects and in experimental animals. European Journal of Endocrinology 153 409-417.

Peake PW, Shen Y, Campbell LV \& Charlesworth JA 2006 Human adiponectin binds to bacterial lipopolysaccharide. Biochemical and Biophysical Research Communications 341 108-115.

Richards AA, Stephens T, Charlton HK, Jones A, Macdonald GA, Prins JB \& Whitehead JP 2006 Adiponectin multimerization is dependent on conserved lysines in the collagenous domain: evidence for regulation of multimerization by alterations in posttranslational modifications. Molecular Endocrinology 20 1673-1687.

Sato C, Yasukawa Z, Honda N, Matsuda T \& Kitajima K 2001 Identification and adipocyte differentiation-dependent expression of the unique disialic acid residue in an adipose tissue-specific glycoprotein, adipo Q. Journal of Biological Chemistry 276 28849-28856.

Skibeli V, Nissen-Lie G \& Torjesen P 2001 Sugar profiling proves that human serum erythropoietin differs from recombinant human erythropoietin. Blood 98 3626-3634.

Suzuki S, Wilson-Kubalek EM, Wert D, Tsao TS \& Lee DH 2007 The oligomeric structure of high molecular weight adiponectin. FEBS Letters 581 809-814.

Tsao TS, Murrey HE, Hug C, Lee DH \& Lodish HF 2002 Oligomerization state-dependent activation of NF-kappa B signaling pathway by adipocyte complement-related protein of $30 \mathrm{kDa}$ (Acrp30). Journal of Biological Chemistry 277 29359-29362.
Tsao TS, Tomas E, Murrey HE, Hug C, Lee DH, Ruderman NB, Heuser JE \& Lodish HF 2003 Role of disulfide bonds in Acrp30/Adiponectin structure and signaling specificity: different oligomers activate different signal transduction pathways. Journal of Biological Chemistry 278 50810-50817.

Waki H, Yamauchi T, Kamon J, Ito Y, Uchida S, Kita S, Hara K, Hada Y, Vasseur F, Froguel $\mathrm{P}$ et al. 2003 Impaired multimerization of human adiponectin mutants associated with diabetes. Molecular structure and multimer formation of adiponectin. Journal of Biological Chemistry 278 40352-40363.

Wang Y, Xu A, Knight C, Xu LY \& Cooper GJ 2002 Hydroxylation and glycosylation of the four conserved lysine residues in the collagenous domain of adiponectin. Potential role in the modulation of its insulin-sensitizing activity. Journal of Biological Chemistry 277 19521-19529.

Wang Y, Lu G, Wong WP, Vliegenthart JF, Gerwig GJ, Lam KS, Cooper GJ \& Xu A 2004 Proteomic and functional characterization of endogenous adiponectin purified from fetal bovine serum. Proteomics 4 3933-3942.

Wang Y, Lam KS, Xu JY, Lu G, Xu LY, Cooper GJ \& Xu A 2005 Adiponectin inhibits cell proliferation by interacting with several growth factors in an oligomerization-dependent manner. Journal of Biological Chemistry 280 18341-18347.

Wang Y, Xu LY, Lam KS, Lu G, Cooper GJ \& Xu A $2006 a$ Proteomic characterization of human serum proteins associated with the fatderived hormone adiponectin. Proteomics 6 3862-3870.

Wang Y, Lam KS, Chan L, Chan KW, Lam JB, Lam MC, Hoo RC, Mak WW, Cooper GJ \& Xu A $2006 b$ Post-translational modifications of the four conserved lysine residues within the collagenous domain of adiponectin are required for the formation of its high molecular weight oligomeric complex. Journal of Biological Chemistry $\mathbf{2 8 1}$ 16391-16400.

Xu A, Chan KW, Hoo RL, Wang Y, Tan KC, Zhang J, Chen B, Lam MC, Tse C, Cooper GJ et al. 2005 Testosterone selectively reduces the high molecular weight form of adiponectin by inhibiting its secretion from adipocytes. Journal of Biological Chemistry $\mathbf{2 8 0}$ 18073-18080.

Received in final form 12 April 2007

Accepted 15 May 2007

Made available online as an Accepted Preprint 18 May 2007 